\title{
Simultaneous release of adenylate kinase and cytochrome $c$ in cell death
}

\section{Dear Editor,}

Cytochrome $c$ (Cyt $c$ ) is located within the mitochondrial intermembrane space, and it is an essential constituent of the respiratory chain. The translocation of Cyt $c$ from mitochondria to the cytosol is an important step in the apoptotic signaling pathway, linking mitochondrial changes to the activation of execution caspases (Liu et al, 1996). Once translocated into the cytosol, Cyt $c$ together with Apaf-1 and procaspase-9 form a multiprotein complex, the apoptosome, which initiates the activation of caspase-3 (Li P et al, 1997). Studies in cell-free sytems have shown that addition of Cyt $c$ to cytosols directly activates caspase-3, whereas immunodepletion of Cyt $c$ from cell homogenates prevents caspase-3 activation. Furthermore, microinjection of Cyt $c$ into intact cells has been shown to induce apoptosis (Li F et al, 1997). Although Cyt $c$ release has been observed in many experimental models of apoptosis, the mechanism of translocation across the outer mitochondrial membrane is unclear. One question is whether Cyt $c$ release occurs by a selective transport mechanism into the cytosol, or by a nonselective change of permeability in the outer mitochondrial membrane. To distinguish between these two possibilities, we monitored the translocation of Cyt $c$ and of the mitochondrial intermembrane space marker adenylate kinase (ADK).

We developed a method that allowed us to separate cytosolic fractions of Jurkat cells from heavy organelles (mitochondria and nuclei) rapidly, and without disturbing mitochondrial membrane integrity. The plasma membrane was selectively permeabilized with digitonin, which strongly binds cholesterol, in a sucrose containing buffer, and soluble cytosolic proteins were separated by rapid centrifugation. To control the selectivity of digitonin permeabilization we assayed the activity of mitochondrial and non-mitochondrial marker enzymes in the supernatant: lactate dehydrogenase (LDH) as cytosolic marker, adenylate kinase (ADK) for the mitochondrial intermembrane space, and citrate synthase and glutamate dehydrogenase as mitochondrial matrix markers. In Jurkat cells, ADK activity is predominantly (ca.75\%) due to the mitochondrial isoform 2. A basal activity in the cytosol is due to the cytosolic isoform 1. Treatment with low concentrations (35 $\mu \mathrm{g} / 4 \times 10^{6}$ cells) of digitonin selectively lysed the cholesterol-rich plasma membrane and released LDH into the supernatant (Figure 1A). Sevenfold higher concentrations were needed to permeabilize the outer mitochondrial membrane (low in cholesterol), as evidenced by the release of the mitochondrial intermembrane proteins Cyt $c$ and ADK. To extract cytosolic samples in apoptosis experiments, we selected a digitonin concentration $\left(35 \mu \mathrm{g} / 4 \times 10^{6}\right.$ cells), which did not damage the outer mitochondrial membrane.
We then used three different apoptosis-inducing compounds, which do not directly interact with mitochondria. Cycloheximide, camptothecin, or actinomycin D elicited $70-90 \%$ apoptosis in Jurkat cells within $7 \mathrm{~h}$ (Figure 1B). Cyt $c$ release from mitochondria preceded nuclear changes, and was paralleled by increasing ADK activity in the cytosolic fractions. Similar results were obtained when apoptosis was triggered by CD95 ligation, which involves the early activation of signaling caspases (Figure 1C). Mitochondrial matrix enzymes were not detected in the cytosol at any time (not shown).

We also investigated the release of mitochondrial intermembrane proteins in a model of necrotic cell death induced by staurosporine (STS) in combination with oligomycin (Leist et al, 1997). Compared to STS-induced apoptosis, necrotic Cyt $c$ release was delayed by about $60 \mathrm{~min}$ (Figure 1C). This delay was also paralleled by a delay in the translocation of the ADK. Thus, the simultaneous appearance of Cyt $c$ and ADK in the cytosol seems to be a phenomenon common to different modes of cell death.

Execution caspases, once activated, may impair the barrier function of mitochondrial membranes as part of their function to make cellular demise irreversible (Green and Kroemer, 1998). Such proteolytic feed-back loops might transform an initially selective Cyt $c$ translocation into a non-selective protein leakage due to the permeabilization of the outer mitochondrial membrane. To test this possibility, we stimulated Jurkat cells with STS in the presence or absence of the caspase inhibitor N-benzyloxycarbonyl-ValAla-Asp-fluoromethylketone (zVAD). Figure 1D shows that the kinetics and the extent of Cyt $c$ release were not influenced by $Z V A D$, and ADK release paralleled Cyt $c$ release under all conditions. Therefore, redistribution of ADK to the cytosol is unlikely to be due to any secondary degradative effect of downstream caspases on the mitochondria, at least in this model. In contrast, upstream caspases were involved in CD95 stimulated Cyt $c$ and ADK release, since ZVAD completely prevented redistribution of both proteins (not shown).

In summary, our data demonstrate that, in dying cells, mitochondria release intermembrane proteins in a nonselective manner. The $26 \mathrm{kD}$ mitochondrial intermembrane protein ADK was released simultaneously to Cyt $c$ in all cases investigated here. A non-selective release of mitochondrial proteins would also be in line with the concomitant release of other apoptogenic factors, such as the $50 \mathrm{kD}$ protein AIF (Green and Kroemer, 1998). The observation that release of both Cyt $c$ and ADK from mitochondria was found also in necrosis further supports our previous suggestion that the two modes of cell death share similarities in the signaling pathway (Leist and 
Nicotera, 1997). A major change in permeability of the outer mitochondrial membrane, which results in the translocation of several proteins would render the mitochondrial alterations irreversible. In most, but not all (Jäättelä et al, 1998), cases the cell would be committed to die, with or without activation of downstream caspases (Green and
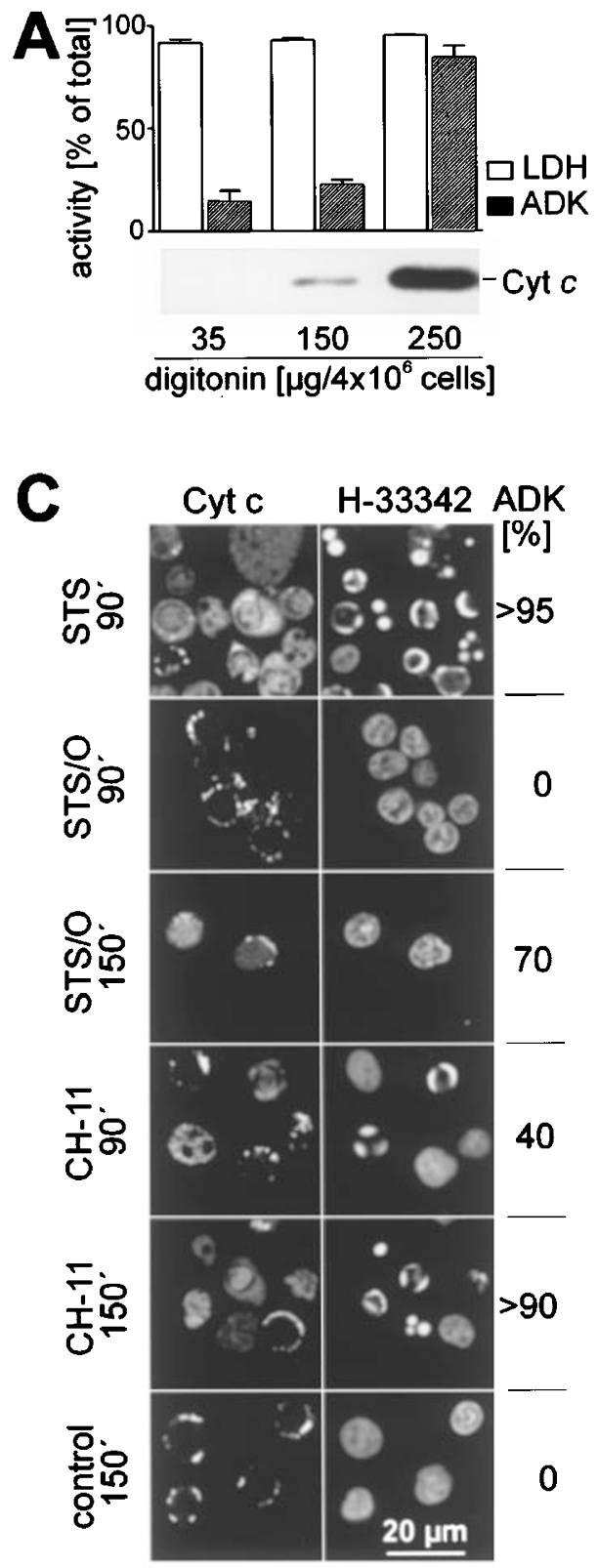
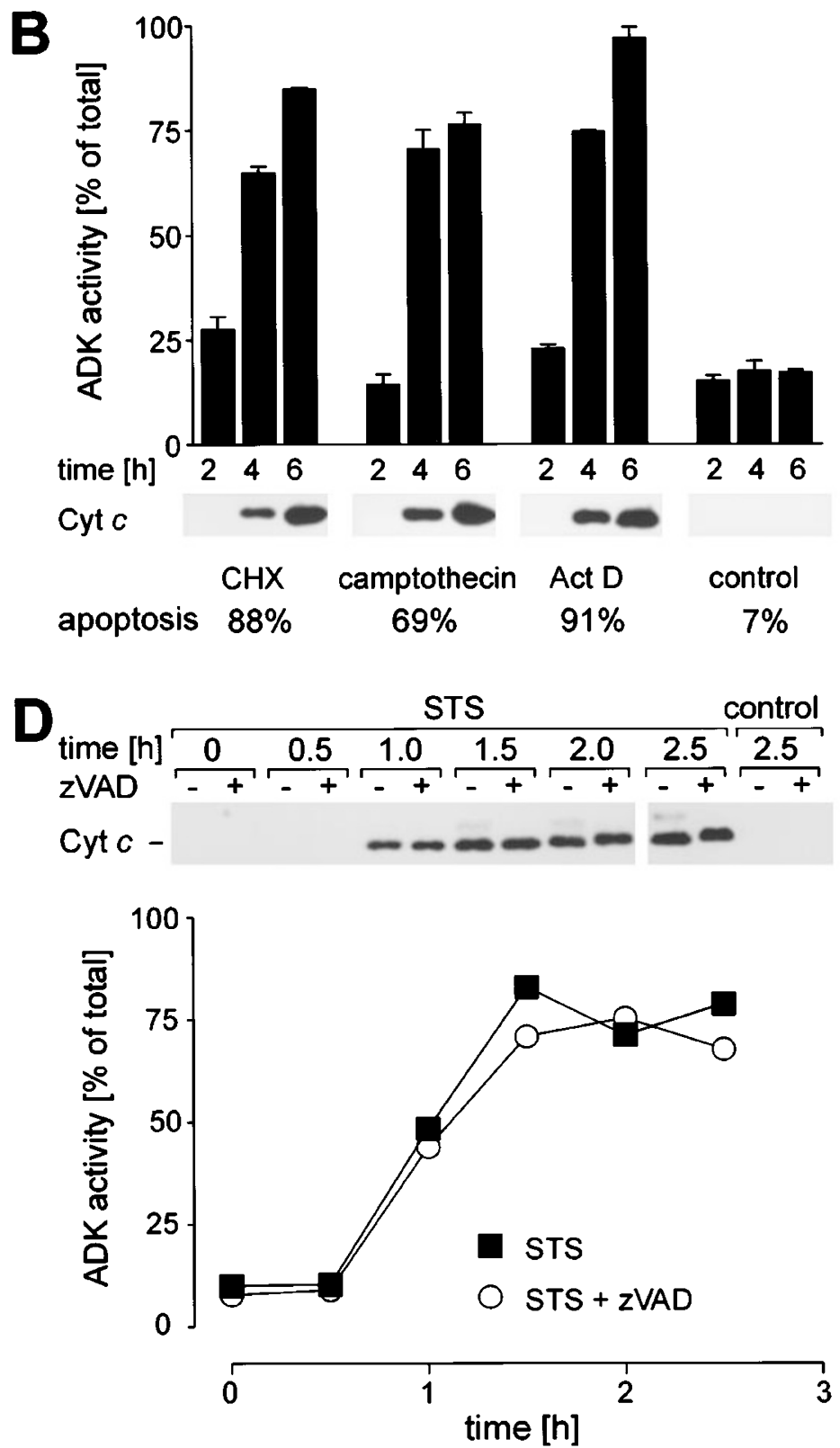

Figure 1 Simultanous release of cytochrome $c($ Cyt $c$ ) and adenylate kinase (ADK). (A) Selective lysis of the plasma membrane for rapid cell fractionation. Jurkat cells were incubated for $30 \mathrm{~s}$ with lysis buffer $\left(75 \mathrm{mM} \mathrm{NaCl}, 1 \mathrm{mM} \mathrm{NaH}_{2} \mathrm{PO}_{4}, 8 \mathrm{mM} \mathrm{Na}_{2} \mathrm{H} \mathrm{PO}_{4}, 250 \mathrm{mM}\right.$ sucrose) containing digitonin. Heavy organelles and cell debris were pelleted for $60 \mathrm{~s}$. The supernatant was analyzed for Cyt $c$ by SDS-PAGE and immunoblotting (Pharmingen), and for lactate dehydrogenase (LDH) and ADK by spectrophotometric determination of the enzyme activities. Activity in lysates obtained after treatment with Triton- $X 100$ were defined as $100 \%$. (B) Release of Cyt $c$ and ADK by different apoptosis inducing compounds. Jurkat cells were exposed to $200 \mu \mathrm{M}$ cycloheximide (CHX), $20 \mu \mathrm{M}$ camptothecin, $2 \mu \mathrm{g} / \mathrm{ml}$ actinomycin D (Act D), or solvent (control). At the times indicated, cytosolic fractions were isolated by selective lysis of the plasma membrane with $35 \mu \mathrm{g}$ digitonin $/ 4 \times 10^{6} \mathrm{cells}$, and analyzed for Cyt $c$ and ADK. The apoptosis rate was determined after $7 \mathrm{~h}$ by scoring cells with condensed chromatin after Hoechst 33342 staining. (C) Analysis of Cyt $c$ release in individual apoptotic or necrotic cells. Cells were incubated with anti CD95 antibody (CH-11, $100 \mathrm{ng} / \mathrm{ml})$, staurosporine (STS, $1 \mu \mathrm{M})$, or a combination of oligomycin $(2.5 \mu \mathrm{M})$ plus STS (STS/O) as described (Leist et al, 1997). After the times indicated, cells were fixed with $4 \%$ paraformaldehyde, immunostained for Cyt $c$ (Pharmingen), counterstained with Hoechst 33342, and imaged by confocal microscopy (Leica TCS-4D). ADK samples were obtained from parallel cultures by digitonin lysis. Data are shown as percentage of the maximal releaseable activity. (D) Release of mitochondrial intermembrane proteins in the presence or absence of caspase inhibition. Jurkat cells were exposed to STS in the presence or absence of $50 \mu \mathrm{M}$ zVAD (N-benzyloxycarbonyl-Val-Ala-Asp-fluoromethylketone). At the times indicated, cytosolic fractions were isolated by selective lysis of the plasma membrane with $35 \mu \mathrm{g}$ digitonin $/ 4 \times 10^{6}$ cells, and analyzed for Cyt $c$ and ADK 
Kroemer, 1998). It remains to be determined whether cytosolic factors are required for permeabilization of the outer mitochondrial membrane, and whether formation of non-specific pores or rupture of the outer mitochondrial membrane underlies release of mitochondrial proteins.

Barbara Single ${ }^{1}$, Marcel Leist ${ }^{1}$, and Pierluigi Nicotera ${ }^{*, 1}$ ${ }^{1}$ Department of Molecular Toxicology, Faculty of Biology, University of Konstanz, D-78457 Konstanz, Germany *corresponding author: email: pierluigi.nicotera@uni-konstanz.de

\section{References}

Green D and Kroemer G (1998) The central executioners of apoptosis: caspases or mitochondria? Trends Cell Biol. 8: 267-271

Jäättelä M, Wissing D, Kokholm K, Kallunki T and Egeblad M (1998) Hsp70 exerts its anti-apoptotic function downstream of caspase-3-like proteases. EMBO J. 17: in press

Leist M and Nicotera P (1997) The shape of cell death. Biochem. Biophys. Res. Commun. 236: $1-9$

Leist M, Single B, Castoldi AF, Kühnle S and Nicotera P (1997) Intracellular adenosine triphosphate (ATP) concentration: a switch in the decision between apoptosis and necrosis. J. Exp. Med. 185: 1481-1486

Li F, Srinivasan A, Wang Y, Armstrong RC, Tomaselli KJ and Fritz LC (1997) Cellspecific induction of apoptosis by microinjection of cytochrome $c-B c l-x_{L}$ has activity independent of cytochrome crelease. J. Biol. Chem. 272: 30299-30305

Li P, Nijhawan D, Budihardjo I, Srinivasula SM, Ahmad M, Alnemri ES and Wang X (1997) Cytochrome $c$ and dATP-dependent formation of Apaf-1/Caspase-9 complex initiates an apoptotic protease cascade. Cell 91: 479-489

Liu X, Kim CN, Yang J, Jemmerson R and Wang X (1996) Induction of apoptotic program in cell-free extracts: requirement for dATP and cytochrome $c$. Cell 86 : $147-157$ 